\title{
Oxymatrine inhibits the proliferation of CaSki cells via downregulating HPV16E7 expression
}

\author{
ZHIJUN PEI $^{1 *}$, JING ZENG $^{2 *}$, YAN GAO $^{1}$, FUYAN LI $^{1}$, WEI LI ${ }^{1}$, \\ HONG ZHOU $^{1}$, YI YANG ${ }^{1}$, RUIMIN WU ${ }^{1}$, YIJIA CHEN ${ }^{1}$ and JIE LIU $^{3}$
}

\begin{abstract}
${ }^{1}$ Department of PET Center and Institute of Anesthesiology and Pain, Taihe Hospital, Hubei University of Medicine, Shiyan, Hubei 442000; ${ }^{2}$ Department of Infection Control, Taihe Hospital, Hubei University of Medicine, Shiyan, Hubei 442000; ${ }^{3}$ Department of Nuclear Medicine, Union Hospital, Tongji Medical College of Huazhong University of Science and Technology, Hubei Province Key Laboratory of Molecular Imaging, Wuhan, Hubei 430022, P.R. China
\end{abstract}

Received January 16, 2016; Accepted March 21, 2016

DOI: 10.3892/or.2016.4800

\begin{abstract}
Treatment of recurrent and metastatic cervical cancer remains a challenge, especially in developing countries, which lack efficient screening programs. HPVI6E7 has been reported to play an important role in the development of cervical cancer. In recent years, oxymatrine, which was traditionally used as anti-malarial agent, has been shown to inhibit tumor growth with low toxicity to normal cells. In the present study, we investigated the mechanisms underlying the antitumor effect of oxymatrine in cervical cancer. The CCK-8 assay was used to compare the proliferation of untreated and oxymatrine-treated cervical cancer CaSki cells. Flow cytometry was applied to observe the effect of oxymatrine on apoptosis and the cell cycle distribution of CaSki cells. We used qRT-PCR and western blot analysis to determine the mRNA level and protein level of HPV16E7. The HPV16E7 siRNA inhibition was also performed to confirm the effect of downregulating HPV16E7 on the proliferation in CaSki cells. Our results revealed that oxymatrine-treated cells showed time-dependent and dose-dependent inhibition of proliferation and a significant increase in apoptosis. Oxymatrine arrested CaSki cells in G0/G1 phase and S phase while decreased the
\end{abstract}

Correspondence to: Dr Jie Liu, Department of Nuclear Medicine, Union Hospital, Tongji Medical College of Huazhong University of Science and Technology, Hubei Province Key Laboratory of Molecular Imaging, Wuhan, Hubei 430022, P.R. China

E-mail: liujiehuazhong@sina.com

${ }^{*}$ Contributed equally

Abbreviations: HPV, human papilloma virus; FBS, fetal bovine serum; PFA, paraformaldehyde; PI, propidium iodide; GAPDH, glyceraldehyde-3-phosphate dehydrogenase; PVDF, polyvinylidenedifluoride

Key words: oxymatrine, cervical cancer, HPV16E7, proliferation, cell cycle, apoptosis cells in $\mathrm{G} 2 / \mathrm{M}$ phase. The expression of HPV16E7 was significantly downregulated in oxymatrine-treated cells compared with control cells. Knock-down of HPV16E7 effectively inhibited the proliferation of CaSki cells. In conclusion, our data suggest that oxymatrine inhibits cervical cancer growth via downregulation of HPV16E7. Oxymatrine can be considered to be a potential preventive and therapeutic target for cervical cancer.

\section{Introduction}

Cervical cancer, which accounts for $8 \%$ of all cancer deaths, is the third most common cancer in women after breast and colorectal cancer $(1,2)$. The human papilloma virus (HPV) is known to be an essential cause of cervical cancer, among which $60 \%$ or more are with HPV type 16 (HPV16) (3). Carcinogenesis by HPV16 is primarily attributed to the continuous expression of viral protein E6/E7. After infection of HPV, the integration of the viral E6/E7 genes occur to the genome of the cervical epithelium, and the continued expression of E6/E7 not only enhances the neoplastic progression to the cervical epithelium, but also drives the cervical cancer cells to malignant phenotype (4-6). There has been convincing evidence provided by previous studies that the overexpression of HPV16E7 is associated with the development of cervical cancer $(3,7)$. Therefore, the HPVI6E7 is considered to be potential therapeutic target for cervical cancer treatment.

Treatment strategies for cervical cancer today are focused on surgical operation or chemo-radiation. Although a number of chemotherapeutic drugs for treating cervical cancer can be used to control the growth of cancer and have shown certain therapeutic efficacy, the strong side-effects limit their application. Besides, only one-third of women with metastatic cervical cancer respond to chemotherapy and this response is short-lived $(1,6)$. Therefore, to develop novel natural substances with curative selectivity for cervical cancer without showing significant toxic effect to normal cells is becoming much more necessary.

There has been a recent focus on the use of Chinese medicinal herbs to treat a number of diseases. Oxymatrine, which is a quinolizidine alkaloid extracted from the root of tradi- 
tional Chinese herbal medicine Sophora japonica (Sophora flavescens Ait), has been found to possess several biological effects such as anti-inflammation, inhibiting immune reaction, anti-virus and antitumor (8-13). Different from the traditional chemotherapy medicine, oxymatrine has been reported to inhibit the proliferation of a number of cancer cells in vitro and also inhibited viral-induced tumor formation in mice, with little influence to some normal cells $(14,15)$.

As far as we know, there are few studies on the application of oxymatrine in the treatment of cervical cancer $(10,16)$. Besides, we found no report concerning the putative relationship between oxymatrine and HPV16E7 in anticancer study. In the present study, we investigated the effect of oxymatrine on cervical cancer cell line CaSki by evaluating cell proliferation, cell apoptosis, cell cycle, the mRNA and protein expression levels of HPV16E7 gene in vitro. The aim of this study was to explore the mechanisms underlying the antitumor effect of oxymatrine on cervical cancer cells and support experimental data for the application of oxymatrine in the prevention and treatment of cervical cancer.

\section{Materials and methods}

Oxymatrine. We purchased oxymatrine from Chia Tai Tianqing Pharmaceutical Group Co., Ltd. (Nanjing, China). Before the application, we tested this oxymatrine and found that its purity is $>99 \%$ indicated by SDS-PAGE analysis.

Cell line and culture. CaSki cells of human cervical carcinoma (American Type Culture Collection, Manassas, VA, USA) were maintained in RPMI-1640 medium (Gibco, Carlsbad, CA, USA) supplemented with $10 \%$ fetal bovine serum (FBS; Gibco) penicillin $(100 \mu \mathrm{g} / \mathrm{ml})$ and streptomycin $(100 \mathrm{U} / \mathrm{ml})$ in $5 \% \mathrm{CO}_{2}$ atmosphere at $37^{\circ} \mathrm{C}$. The cells were passaged every 2-4 days to keep a proper density and cells in the logarithmic growth phase were used in the experiment.

Proliferation assay. CaSki cells in logarithmic growth phase were seeded in 96-well plates with $1 \times 10^{5}$ cells each well, and cultured cells with RPMI-1640 complete medium. Twentyfour hours later, the medium was replaced with RPMI-1640 complete medium with various concentrations of oxymatrine $(2,4$ and $6 \mathrm{mg} / \mathrm{ml})$ and cultured continuously. In addition, control cells were incubated with medium only. After exposure to oxymatrine, the proliferation of CaSki cells was assessed by using Cell Counting kit-8 assay. After 24, 48 and 72 h, each well of cells were treated with $10 \mu \mathrm{l}$ of Cell Counting kit- 8 solution (Dojindo Laboratories, Kumamoto, Japan) and incubated for $1 \mathrm{~h}$ at $37^{\circ} \mathrm{C}$. Spectrometric absorbance was measured at $450 \mathrm{~nm}$ by an enzyme immunoassay analyzer (Bio-Rad Laboratories, Hercules, CA, USA).

Colony formation assay. Colony formation assay was performed to observe the effect of oxymatrine on colony formation ability of CaSki cells. Cells $\left(1 \times 10^{3}\right)$ were plated in each well of 6-well plate and cultured with RPMI-1640 complete mediumin a humidified atmosphere with $5 \% \mathrm{CO}_{2}$ at $37^{\circ} \mathrm{C}$. After 2 weeks, the colonies were fixed by $4 \%$ paraformaldehyde (PFA) for $15 \mathrm{~min}$ and stained with Giemsa for $20 \mathrm{~min}$. Cell aggregate consisting of 50 or more cells were defined as one colony. Clonies were manually counted by microscope (Olympus, Tokyo, Japan) with x40 field.

Apoptosis assay. Apoptosis assay kit (Multi Sciences, Harbin, China) was used to treat the cell samples and the procedure followed the instructions of the kit. CaSki cells were treated with various concentrations of oxymatrine $(0,2,4$ and $6 \mathrm{mg} / \mathrm{ml})$ for $72 \mathrm{~h}$, and the cells were collected, washed, successively incubated with $5 \mu \mathrm{l}$ of Annexin V-FITC and $5 \mu \mathrm{l}$ of propidium iodide (PI) for $15 \mathrm{~min}$ at room temperature. Before being subjected to flow cytometric analysis, apoptosic ratio of fixed cell sample was measured by flow cytometry (FACSCalibur ${ }^{\mathrm{TM}}$; BD Biosciences, San Jose, CA, USA).

Cell cycle analysis. CaSki cells were treated with different concentrations of oxymatrine $(0,2,4$ and $6 \mathrm{mg} / \mathrm{ml})$ for $72 \mathrm{~h}$. The cells were harvested by trypsinization, then were fixed with $70 \%$ ethanol and resuspended in $20 \mathrm{mg} / \mathrm{Ml}$ PI for $30 \mathrm{~min}$. Flow cytometer (FACSCalibur ${ }^{\mathrm{TM}}$; BD Biosciences) was applied to detect DNA content. Based on the flow cytometry data, we determined the relative proportions of cells in the individual cell-cycle phase fraction.

Quantitative real-time PCR. Total RNA was extracted from CaSki cells which had been treated with $4 \mathrm{mg} / \mathrm{ml}$ of oxymatrine for $72 \mathrm{~h}$ or the control cells using TRIzol RNA reagent (Invitrogen, Carlsbad, CA, USA), and then was treated with DNAse I (Roche, Basel, Switzerland) to remove contaminating genomic and adenoviral DNA. MMLV reverse transcriptase kit (Promega, Madison, WI, USA) was used to convert the prepared total RNA to cDNA, which was then subjected to quantitative real-time PCR (qRT-PCR) using a SYBR-Green Master Mix kit on a Bio-Rad connect real-time PCR platform. Taq DNA polymerase was used. Glyceraldehyde-3-phosphate dehydrogenase (GAPDH) was used to normalize the mRNA levels. The sequences of primers were as follows: HPV16E7 (157 bp), sense: 5'-ATGCATGGAGATACACCT-3' and antisense: 5'-TTATGGTTTCTGAGAACA-3'; GAPDH (101 bp), sense: 5'-ACAACTTTGGTATCGTGGAAGG-3' and reverse, 5'-GCCATCACGCCACAGTTTC-3'. Data were analyzed using the $2^{-\Delta \Delta \mathrm{Ct}}$ method.

Western blot analysis. The protein was extracted from CaSki cells treated with $4 \mathrm{mg} / \mathrm{ml}$ of oxymatrine for $72 \mathrm{~h}$ and the control cells. The cells were lysed by lysis buffer $(150 \mathrm{mM}$ $\mathrm{NaCl}, 0.1 \%$ SDS, $0.5 \%$ sodium deoxycholate, $1 \%$ Nonidet P-40 and $50 \mathrm{mM}$ Tris, $\mathrm{pH} 8.0$ ), with the addition of $2 \mathrm{mM}$ phenylmethylsulfonyl fluoride, and then centrifuged at $12,000 \mathrm{x} \mathrm{g}$ for $30 \mathrm{~min}$ at $4^{\circ} \mathrm{C}$. Protein concentrations were determined by protein assay kit (Sigma, St. Louis, MO, USA). Equal amounts of proteins $(20 \mu \mathrm{g})$ were boiled for $10 \mathrm{~min}$, and loaded onto a $15 \%$ SDS-PAGE gel and then transferred to the polyvinylidenedifluoride (PVDF) membranes. The membranes were incubated for $1 \mathrm{~h}$ with blocking buffer (PBS with 5\% skim milk and $0.1 \%$ Tween-20) and then with anti-HPV16E7 primary antibody (Abcam, Cambridge, MA, USA) overnight at $4^{\circ} \mathrm{C}$. Secondary antibody (Abcam) was used to incubate the membranes for $1 \mathrm{~h}$ at room temperature. Before each step, membranes were washed 3 times by PBST. The protein bands were visualized by enhanced chemiluminescence. We used 

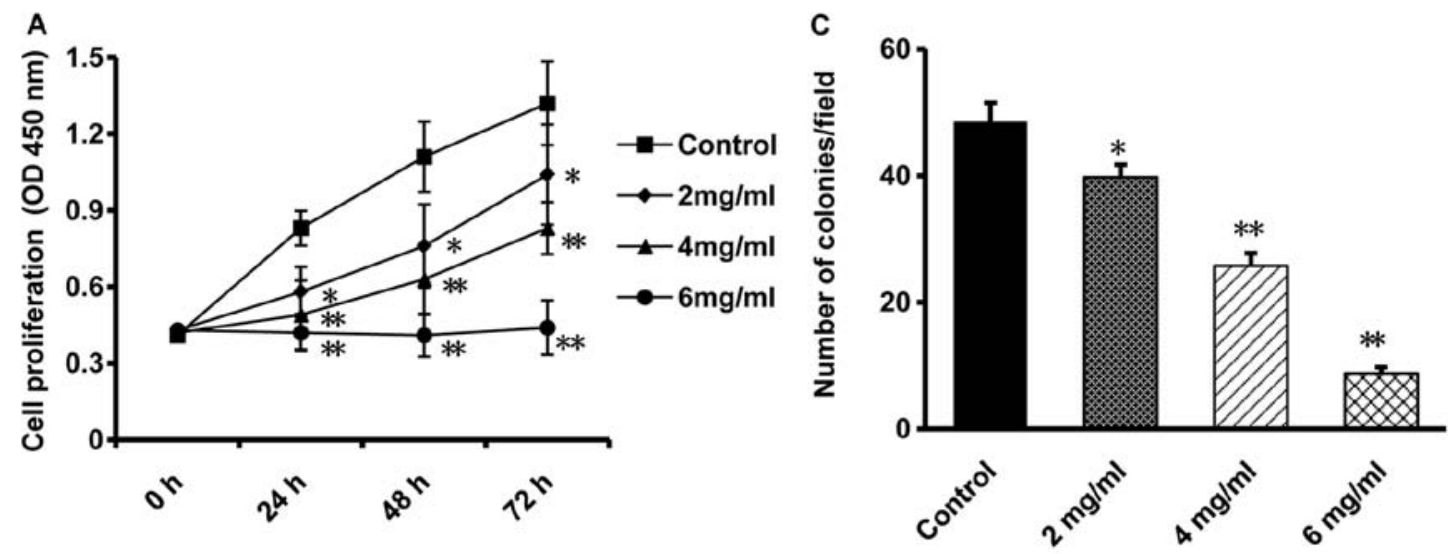

B
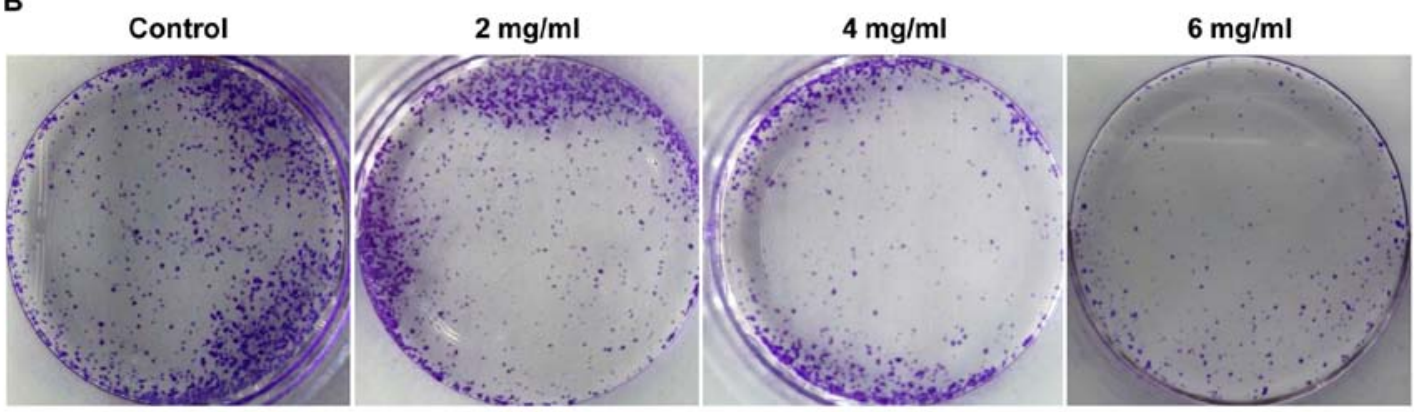

Figure 1. Oxymatrine inhibits the growth of CaSki cells. (A) CaSki cells were treated with oxymatrine at different concentrations (2,4 and $6 \mathrm{mg} / \mathrm{ml})$ seperately for various time. CCK-8 assay was used and the OD $450 \mathrm{~nm}$ values showed that oxymatrine could inhibit the proliferation of CaSki cells in both dosedependent and time-dependent manner. (B) Representative images of colony forming assay for oxymatrine treatment group and the control group. (C) Colony formation assay results showed that the number of CaSki colonies in the oxymatrine group was obviously reduced, compared with the other groups and the suppressing effect was dose-dependent. " $\mathrm{P}<0.05$ and ${ }^{* *} \mathrm{P}<0.01$, respectively, both indicating that a significant difference between the control group and the oxymatrine treated groups.

$\beta$-actin (Santa Cruz Biotechnology, Santa Cruz, CA, USA) as the internal control.

RNA interference. The siRNA for HPV16E7 was designed and synthesized by Sangon Biotech Co., Ltd., (Shanghai, China), and the sequences were as follows (5'-3'): GCACACACGTA GACATTCGdTdT; (3'-5'): dTdTCGUGUGUGCAUCUGUA AGC. A scrambled siRNA which had no homology with human genome was also produced for use as a negative control. The siRNA duplex (50 $\mathrm{nM})$ was transfected using Lipofectamine 2000 reagent (Invitrogen) following the instructions of the manufacturer. The CaSki cells were effected by HPV16E7 siRNA for $72 \mathrm{~h}$ before the analysis of cell proliferation and HPVI6E7 expression.

Tumorigenicity assay. The ethics approval for this protocol was obtained from The Institutional Animal Care and Use Committee at Hubei University of Medicine. CaSki cells treated with $4 \mathrm{mg} / \mathrm{ml}$ of oxymatrine for $72 \mathrm{~h}$ and control CaSki cells were injected subcutaneously into 4 -week old nude mice $\left(3 \times 10^{6}\right.$ cells/mouse), respectively. Four weeks after the injection, mice were sacrificed by injecting excessive chloral hydrate and tumors were separated and weighed.

Statistical analysis. All experiments were performed for at least three times, and data are shown as the mean \pm SD. One-way ANOVA was used to determine statistically significant differences between groups, and $\mathrm{P}<0.05$ was considered statistically significant. Analyses were carried out using GraphPad Prism version 5.0 (Graphpad Software, Inc., La Jolla, CA, USA).

\section{Results}

Oxymatrine inhibits proliferation and clonogenicity of CaSki cells. CaSki cells were treated with various concentrations of oxymatrine $(0,2,4$ and $6 \mathrm{mg} / \mathrm{ml})$ for 24, 48 and $72 \mathrm{~h}$. CCK-8 assay was performed to determine the effect of oxymatrine on cell proliferation and found a time-dependent and concentration-dependent inhibition of oxymatrine to the growth of CaSki cells, compared with that in the control cells $(\mathrm{P}<0.05$, $\mathrm{n}=6$; Fig. 1A). Especially when the CaSki cells were treated with oxymatrine for $72 \mathrm{~h}$, the inhibitory effect was much more significant than that treated for $24 \mathrm{~h}(\mathrm{P}<0.05, \mathrm{n}=6$; Fig. 1A). Therefore, we chose the time-point of $72 \mathrm{~h}$ for further investigation.

The inhibitory effect of oxymatrine on CaSki cells was then confirmed by colony formation assay. The results showed that after treated with concentrations of oxymatrine $(0,2,4$ and $6 \mathrm{mg} / \mathrm{ml}$ ), the colony formation ability of CaSki cells was significantly decreased in a dose-dependent manner, compared with the control group $(\mathrm{P}<0.05, \mathrm{n}=6$; Fig. 1B and $\mathrm{C})$, indicating that oxymatrine is able to inhibit the growth of cervical cancer cells.

Oxymatrine induces apoptosis to CaSki cells. The effect of oxymatrine on cell death of CaSki cells was investigated by 

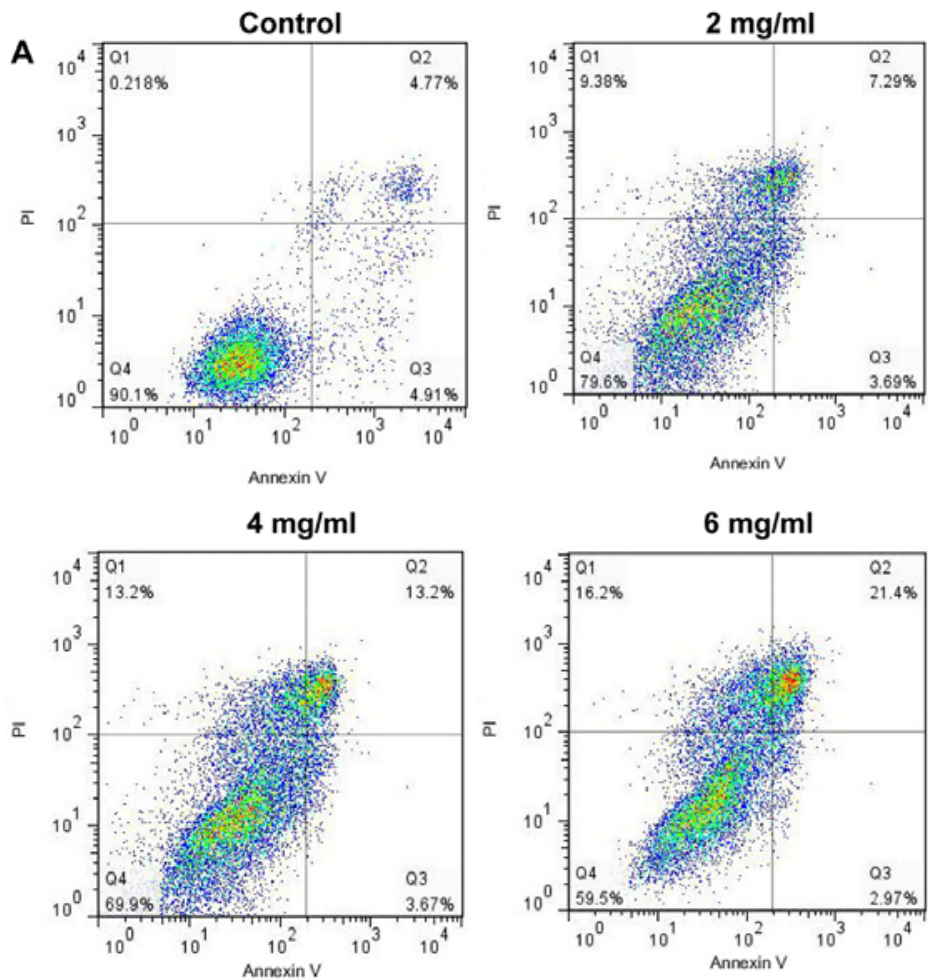

B

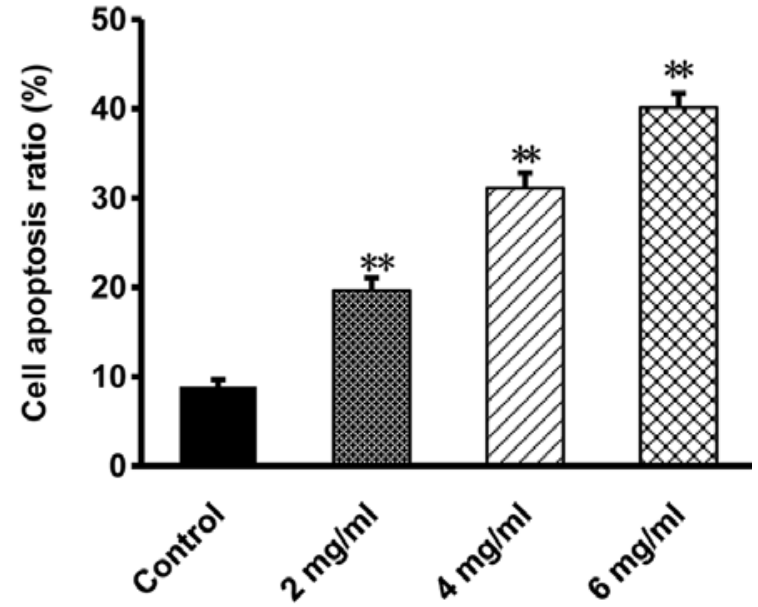

Figure 2. Oxymatrine induces apoptosis to CaSki cells. (A) Representative images of cell apoptosis analysis using Annexin V-FITC/PI with FCM for the oxymatrine treatment group and the control group. (B) Oxymatrine induced cell apoptosis of CaSki cells in a dose-dependent manner, compared with that in the control group. ${ }^{* *} \mathrm{P}<0.01$, indicating a significant difference between the control group and the oxymatrine treated groups.

A
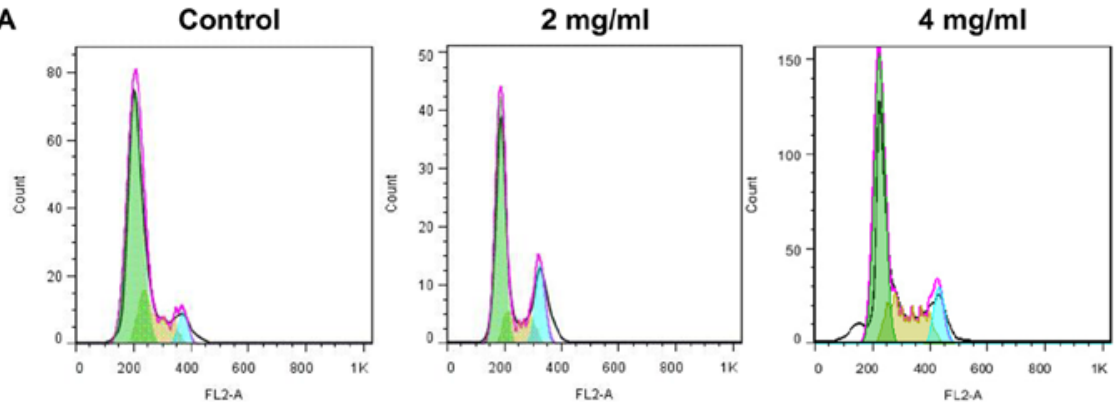

B

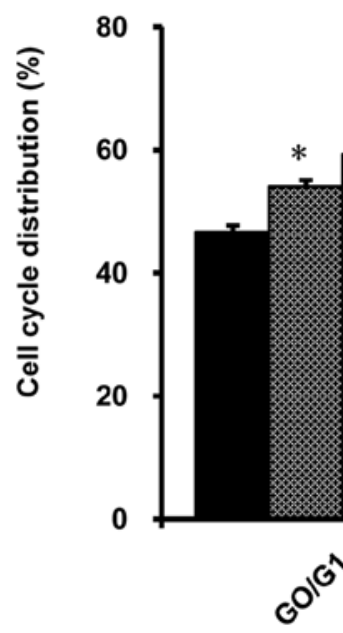

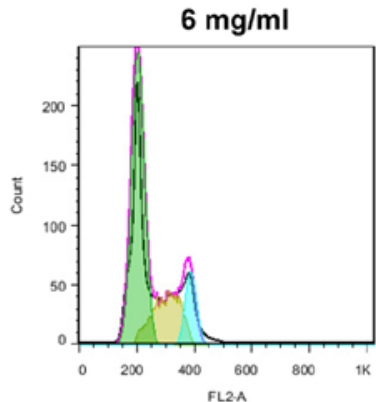

- Control

종 $2 \mathrm{mg} / \mathrm{ml}$

口 $\mathrm{mg} / \mathrm{ml}$

$06 \mathrm{mg} / \mathrm{ml}$

Figure 3. Oxymatrine changed the cell cycle of CaSki cells. (A) Representative images of cell cycle analysis using FCM for oxymatrine treatment group and the control group. (B) After treated with 2, 4 and $6 \mathrm{mg} / \mathrm{ml}$ oxymatrine, the proportion of CaSki cells in G0/G1 phase was prominently increased while the proportion in G2/M phase was decreased, compared with the control group. Besides, the proportion of CaSki cells in S phase was also significantly increased, in the $6-\mathrm{mg} / \mathrm{ml}$ oxymatrine treatment group. ${ }^{*} \mathrm{P}<0.05$ and ${ }^{* *} \mathrm{P}<0.01$, respectively, both indicating a significant difference between the control group and the oxymatrine treated groups. 
A

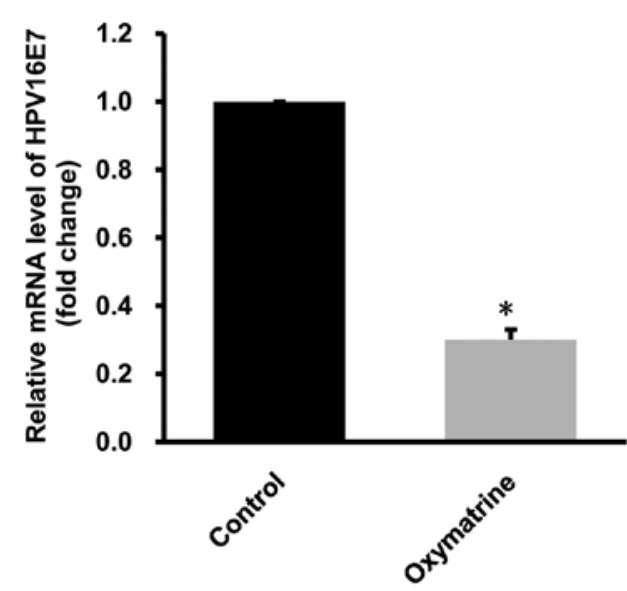

C

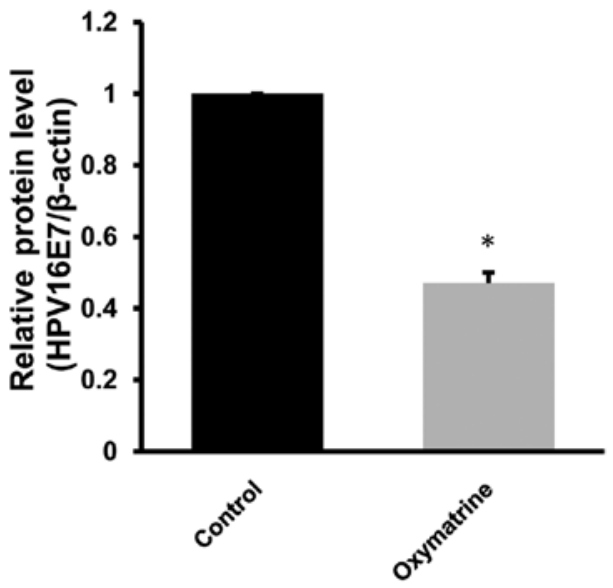

B

Control

oxymatrine

HPV16E7

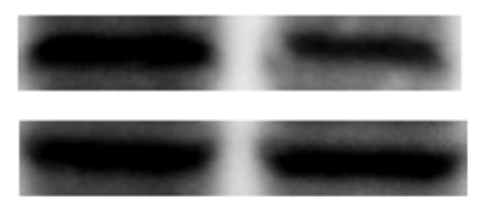

$11 \mathrm{kDa}$

$\beta$-actin

$43 \mathrm{kDa}$

Figure 4. Oxymatrine downregulated the mRNA and protein level of HPV16E7 in CaSki cells. (A) The mRNA level of HPV16E7 was detected by qRT-PCR. The mRNA level of HPV16E7 was normalized to that of GAPDH. (B) Representative images of western blot protein gels for oxymatrine treatment group and the control group. (C) The protein level of HPV16E7 in the oxymatrine treatment group and the control group. ${ }^{* *} \mathrm{P}<0.01$, indicating a significant difference between the control group and the oxymatrine treated group.

evaluating the rate of apoptosis with flow cytometry. Results showed that after treatment with 2,4 and $6 \mathrm{mg} / \mathrm{ml}$ of oxymatrine for $72 \mathrm{~h}$, the rate of CaSki apoptosis was 19.62 \pm 1.71 , $31.84 \pm 2.46$ and $40.94 \pm 2.39 \%$, respectively. The apoptosis rate of the control group was $9.64 \pm 0.98 \%$. In contrast with the control group, the apoptosis rate of oxymatrine treated groups increased significantly in a dose-dependent manner $(\mathrm{P}<0.01, \mathrm{n}=6$; Fig. 2). As the results show, oxymatrine may suppress CaSki cell proliferation by causing cell death.

Oxymatrine changes the cell cycle distribution of CaSki cells. We performed FCM analysis to evaluate the effect of oxymatrine on the cell cycle of CaSki cells. After treatment with 2,4 and $6 \mathrm{mg} / \mathrm{ml}$ of oxymatrine for $72 \mathrm{~h}$, the proportion of $\mathrm{G} 0 / \mathrm{G} 1$ phase was $54.91 \pm 2.08,59.35 \pm 2.17$ and $65.07 \pm 3.22 \%$, respectively, while the percentage of G0/G1 phase control group was $44.35 \pm 1.78 \%$. Compared with that in the control group, CaSki cells of the treated group in G0/G1 phase were markedly increased in a dose-dependent manner, while the cells in $\mathrm{G} 2 / \mathrm{M}$ phase were decreased in the same way $(\mathrm{P}<0.01$, $\mathrm{n}=6$; Fig. 3). Besides, we found that $6 \mathrm{mg} / \mathrm{ml}$ oxymatrine could significantly increase the number of CaSki cells in $\mathrm{S}$ phase, compared to the control cells $(\mathrm{P}<0.01, \mathrm{n}=6$; Fig. 3$)$. The results demonstrate that oxymatrine arrested CaSki cells in G0/G1 phase and $\mathrm{S}$ phase.

Oxymatrine downregulates the $m R N A$ and protein levels of HPVI6E7 in CaSki cells. We performed qRT-PCR and western blot analysis to examine the effect of oxymatrine on the expression of HPV16E7 gene. The results showed that after treated with $4 \mathrm{mg} / \mathrm{ml}$ of oxymatrine, mRNA levels of HPV16E7 gene in the CaSki cells was significantly decreased by $64.5 \pm 2.8 \%(\mathrm{P}<0.01, \mathrm{n}=6$; Fig. $4 \mathrm{~A})$ and protein level was prominently decreased by $52.3 \pm 3.2 \%(\mathrm{P}<0.01, \mathrm{n}=6$; Fig. $4 \mathrm{~B}$ and C), compared with the control group. These data demonstrate that oxymatrine could markedly downregulate the expression of HPV16E7 in CaSki cells.

HPVI6E7 siRNA suppresses the proliferation of CaSki cells. We used HPV16E7 siRNA to knock down HPV16E7 mRNA level to investigate the effect of downregulating HPVI6E7 on the proliferation of CaSki cells. Our results show that after treated with HPV16E7 siRNA, the mRNA and protein level of HPV16E7 in CaSki were reduced by $66.4 \pm 2.7$ and $57.2 \pm 6.1 \%$ respectively, in contrast with the control group treated with scramble siRNA (Fig. 5A-C). After treated with HPV16E7 siRNA, the proliferation of CaSki cells was suppressed in a time-dependent manner, compared with control siRNA $(\mathrm{P}<0.05, \mathrm{n}=6$; Fig. 5D). Our data demonstrate that knocking down HPV16E7 expression could suppress the proliferation of CaSki cells.

Oxymatrine suppressed the growth of CaSki cells in vivo. CaSki cells $\left(3 \times 10^{6}\right)$ treated with oxymatrine or control cells were injected subcutaneously into each athymic nude mouse. Four weeks after the injection, mice were sacrificed and tumors were separated. As showed in the tumor images, all 3 mice injected with control CaSki cells formed xenografts. In contrast, 2 out of 3 mice injected with oxymatrine-treated CaSki cells formed smaller xenografts. The weight of tumors from oxymatrine treated CaSki cells was significantly lower than those from the control CaSki cells $(\mathrm{P}<0.05, \mathrm{n}=3$; Fig. 6B). The results suggest that oxymatrine could suppress tumorigenicity of cervical cancer cells in vivo. 


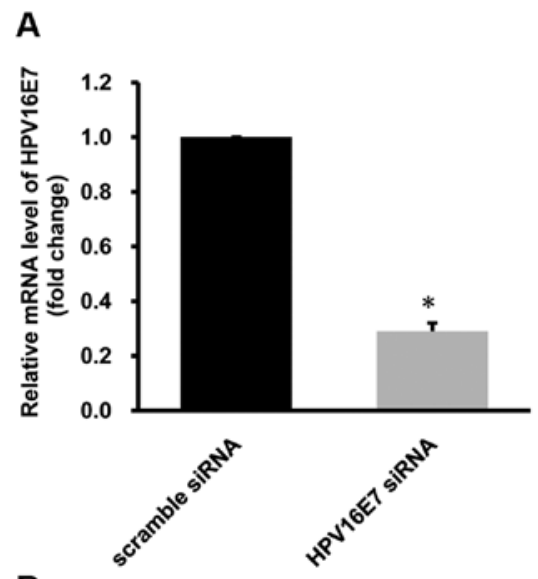

B

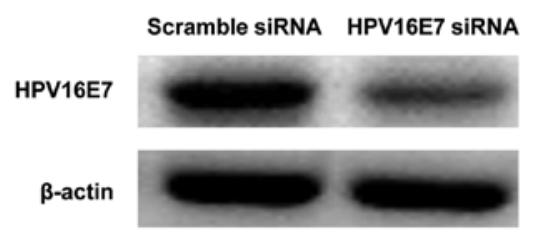

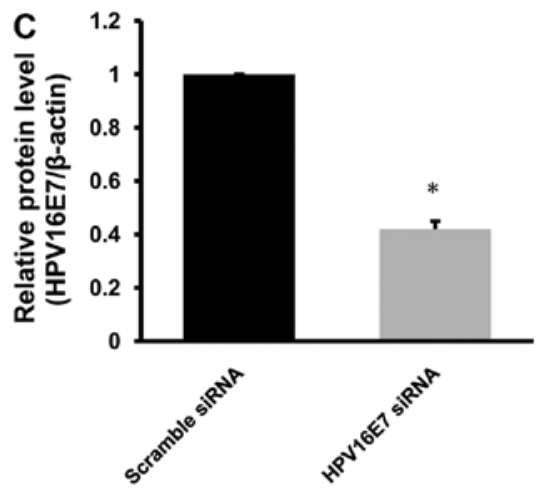

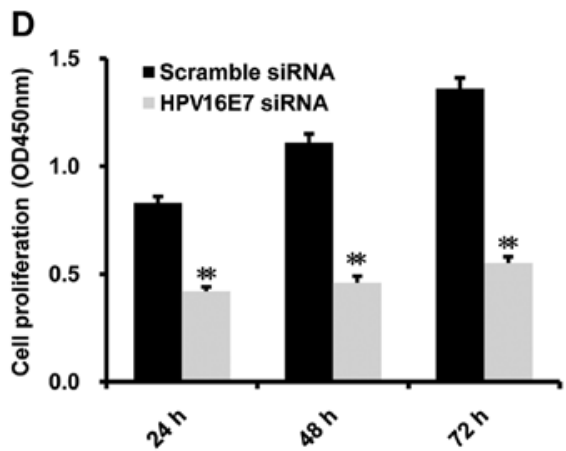

Figure 5. HPV16E7 siRNA downregulates the expression of HPV16E7 and suppresses proliferation in CaSki cells. (A) The mRNA level of HPV16E7 was detected by qRT-PCR. mRNA expression of HPV16E7 was normalized to that of GAPDH. (B) Representative images of western blot protein gels for HPV16E7 siRNA treatment group and the scramble control group. (C) The protein level of HPV16E7 in the HPV16E7 siRNA treatment group and the scramble control group. (D) CCK-8 assay showed when treated with HPV16E7 siRNA for 24 and $48 \mathrm{~h}$, the proliferation of CaSki cells was significantly suppressed, compared with the scramble control group. ${ }^{* *} \mathrm{P}<0.01$, indicating a significant difference between the scramble control group and the HPV16E7 siRNA group.
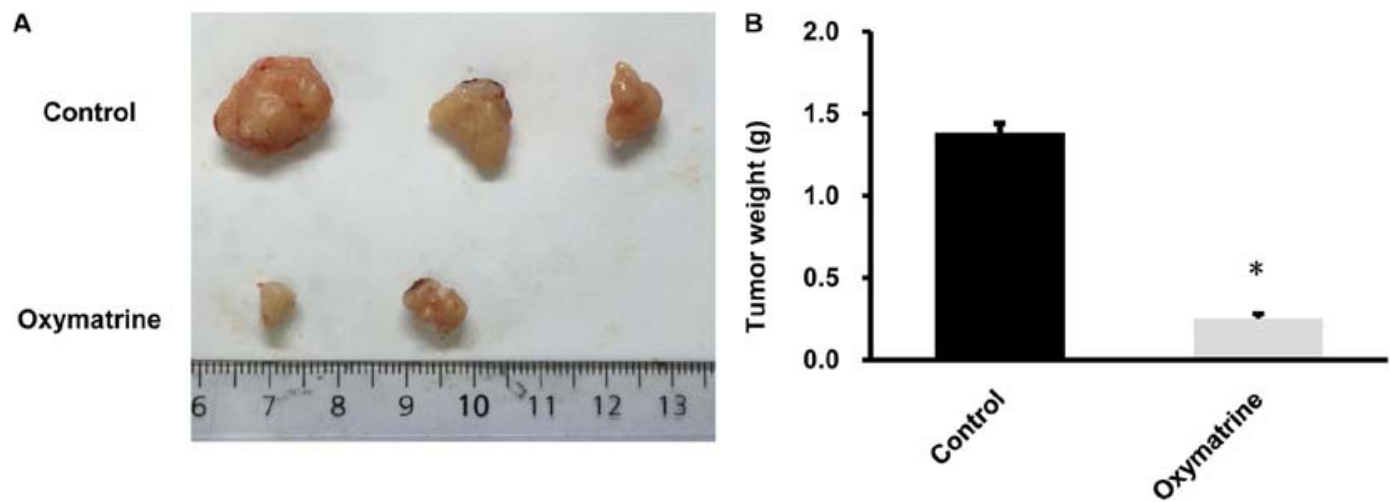

Figure 6. Oxymatrine inhibits the proliferatin of CaSki cells in vivo. (A) The separated tumors derived from oxymatrine treated CaSki cells and the control cells. (B) The weight of tumors of oxymatrine treatment group was significantly lower than that of the control group. ${ }^{*} \mathrm{P}<0.05$, indicating a significant difference between the control and the oxymatrine treated group.

\section{Discussion}

Oxymatrine is a major alkaloid component found in the roots of Sophora species (17). It is commonly used for the treatment of liver disorders and other diseases such as arrhythmia, eczema and skin disorders, leukopenia and bronchitis $(13,18-21)$. Some studies have also reported oxymatrine showed anticancer activity in human gastric cancer cells, pancreatic, ovarian cancer, and human breast cancer cells $(14,16,22)$. However, to the best of our knowledge, the mechanisms of the antitumor effect of oxymatrine on cervical cancer have not yet been elucidated. In the present study, various concentrations of oxymatrine were used to treat cervical cancer cell line CaSki cells, and we found that oxymatrine treatment could induce inhibition of proliferation and apoptosis in cervical cancer CaSki cells in vitro and in vivo.

One of the main reasons for tumorigenesis is loss of control of cell proliferation, which is involved both in tumor initiation and progression (23). Therefore, we applied CCK-8 assay and found that oxymatrine could obviously inhibit cell proliferation in cervical cancer CaSki cells in a time-dependent and dose-dependent manner in vitro. Besides, our colony formation assay results also showed similar inhibition effect of oxymatrine on the colony forming ability of CaSki cells in 
a dose-dependent manner. In vivo, we performed analysis of tumorigenesis and found that the weight of tumors resulted from CaSki cells treated with oxymatrine were significantly reduced, compared with the tumors derived from control CaSki cells. These data indicate that oxymatrine can suppress the growth of cervical cancer cells in vitro and in vivo.

Apoptosis is the process of cell death, with a series of cellular and molecular changes, such as phosphatidylserine externalization, chromatin condensation and cell shrinkage. Apoptosis plays important functions in organ development, homeostasis and immune defense. Uncontrolled cell death is another main reason for tumorigenesis $(24,25)$. Therefore, inducing cell apoptosis of tumor cells is always of great importance in developing anticancer treating methods. In the present study, flow cytometry was applied to the effect of oxymatrine on the apotosis of CaSki cells and the results demonstrated that CaSki cells could be induced to apotosis by oxymatrine in a dose-dependent manner.

Since the change of cell proliferation and apotosis is intimately correlated with the change of the cell cycle $(23,24)$. In this study, in order to investigate the cell cycle related mechanism of the effect of oxymatrine on the proliferation and apotosis on CaSki cells, we also performed flow cytometry and found that the oxymatrine treated CaSki cells were significantly blocked in G0/G1 phase. After treated with increasing concentration of oxymatrine, CaSki cells in G0/G1 phase were prominently increased while cells in the $\mathrm{G} 2 / \mathrm{M}$ phase were obviously decreased, in contrast with control CaSki cells. Moreover, we found that oxymatrine could increase the number of CaSki cells in S phase to a certain extent, and $6 \mathrm{mg} /$ $\mathrm{ml}$ made a significant difference compared to the control cells. When arrested in G0/G1 and S phase, the CaSki cells tend to decrease the proliferation rate with more apoptosis, which is also in accordance with our proliferation and apoptosis assay data. These data suggest that the growth inhibition and apoptosis induction of CaSki cells may result from the effect of oxymatrine on arresting CaSki cells in G0/G1 phase.

According to previous studies, over $60 \%$ of all cervical cancers are closely correlated with HPV16, which encodes the essential genes for virus replication, E6 and E7 oncogenes (7). The viral protein $E 7$ can cause destabilization and the disruption of $R b / E 2 F$ complexes, which is essential for driving the process of cell cycle into $S$ phase, and upregulating the anti-apoptotic protein $\mathrm{Bcl}-2$ to promote cell survival and proliferation $(3,26)$. Several researches have reported that inhibition of the expression of $E 7$ oncogene was efficient in HPV-associated cancer therapy $(15,27,28)$. It is reported by previous studies that oxymatrine showed anti-hepatitis virus effects (29-31). Although no report on the anti-HPV effect was found, our results demonstrated that oxymatrine could decrease both the mRNA and protein levels of HPV16E7. Since the expression of HPV16E7 was closely correlated with the proliferation and apoptosis in CaSki cells, we speculated that oxymatrine could inhibit proliferation and enhance apoptosis in CaSki cells via downregulating HPV16E7. We also used HPV16E7 siRNA to knock-down HPV16E7 expression, and the results indicated that downregulating HPV16E7 expression could suppress the growth of CaSki cells, which was in accordance with our speculation. We also hypothesized that oxymatrine is able to treat cervical cancer through the anti- $H P V$ effect, which is going to be the subject of our further investigation.

In conclusion, our data showed that oxymatrine significantly induced cell apoptosis, and suppressed cell growth of cervical cancer CaSki cells both in vitro and in vivo. Oxymatrine could decrease the CaSki cells in G2/M phase and arrest the CaSki cells in G0/G1 and S phase, which partly mediated proliferation inhibition and apoptosis induction. Besides, we a found that oxymatrine could downregulate the expression of HPV16E7 at the mRNA and protein level, which offered the molecular explanation to the cell growth inhibitory effect of oxymatrine on CaSki cells. Therefore, we believe that oxymatrine can be developed to a potential preventive and therapeutic candidate for cervical cancer treatment. Our data may provide theoretical support for the clinical anticancer application with oxymatrine. However, the toxicity of oxymatrine and its effect on the proliferation of normal cells were not assessed in the present study. In future, we will continue our study on the toxicity of oxymatrine and other effects of oxymatrine in carcinoma cells in vivo and in vitro.

\section{Acknowledgements}

The present study was supported by the National Natural Science Foundation of China (grant no. 81401447).

\section{References}

1. Aggarwal P: Cervical cancer: Can it be prevented? World J Clin Oncol 5: 775-780, 2014.

2. Wang KL: Human papillomavirus and vaccination in cervical cancer. Taiwan J Obstet Gynecol 46: 352-362, 2007.

3. Haedicke $\mathbf{J}$ and Iftner $\mathrm{T}$ : Human papillomaviruses and cancer. Radiother Oncol 108: 397-402, 2013.

4. Almajhdi FN, Senger T, Amer HM, Gissmann L and Öhlschläger P: Design of a highly effective therapeutic HPV16 E6/E7-specific DNA vaccine: Optimization by different ways of sequence rearrangements (shuffling). PLoS One 9: e113461, 2014.

5. Chen S, Liao C, Lai Y, Fan Y, Lu G, Wang H, Zhang X, Lin MC, Leng S and Kung HF: De-oncogenic HPV E6/E7 vaccine gets enhanced antigenicity and promotes tumoricidal synergy with cisplatin. Acta Biochim Biophys Sin (Shanghai) 46: 6-14, 2014.

6. Duenas-Gonzalez A, Serrano-Olvera A, Cetina L and Coronel J: New molecular targets against cervical cancer. Int J Womens Health 6: 1023-1031, 2014.

7. Boulet GA, Horvath CA, Berghmans S and Bogers J: Human papillomavirus in cervical cancer screening: important role as biomarker. Cancer Epidemiol Biomarkers Prevy 17: 810-817, 2008.

8. Fei ZW, Qiu MK, Qi XQ, Dai YX, Wang SQ, Quan ZW, Liu YB and Ou JM: Oxymatrine suppresses proliferation and induces apoptosis of hemangioma cells through inhibition of HIF-1a signaling. Int J Immunopathol Pharmacol 28: 201-208, 2015.

9. Guo B, Zhang T, Su J, Wang K and Li X: Oxymatrine targets EGFR(p-Tyr845) and inhibits EGFR-related signaling pathways to suppress the proliferation and invasion of gastric cancer cells. Cancer Chemother Pharmacol 75: 353-363, 2015.

10. Li M, Su BS, Chang LH, Gao Q, Chen KL, An P, Huang C, Yang J and Li ZF: Oxymatrine induces apoptosis in human cervical cancer cells through guanine nucleotide depletion. Anticancer Drugs 25: 161-173, 2014

11. Wang B, Han Q and Zhu Y: Oxymatrine inhibited cell proliferation by inducing apoptosis in human lung cancer A549 cells. Biomed Mater Eng 26 (Suppl 1): S165-S172, 2015

12. Zhang Y, Sun S, Chen J, Ren P, Hu Y, Cao Z, Sun H and Ding Y: Oxymatrine induces mitochondria dependent apoptosis in human osteosarcoma MNNG/HOS cells through inhibition of PI3K/Akt pathway. Tumour Biol 35: 1619-1625, 2014. 
13. Guzman JR, Koo JS, Goldsmith JR, Mühlbauer M, Narula A and Jobin C: Oxymatrine prevents NF- $\mathrm{KB}$ nuclear translocation and ameliorates acute intestinal inflammation. Sci Rep 3: 1629, 2013

14. Wang W, You RL, Qin WJ, Hai LN, Fang MJ, Huang GH, Kang RX, Li MH, Qiao YF, Li JW, et al: Anti-tumor activities of active ingredients in Compound Kushen Injection. Acta Pharmacol Sin 36: 676-679, 2015.

15. Ying XJ, Jin B, Chen XW, Xie J, Xu HM and Dong P: Oxymatrine downregulates HPV16E7 expression and inhibits cell proliferation in laryngeal squamous cell carcinoma Hep-2 cells in vitro. BioMed Res Int 2015: 150390, 2015.

16. Sun M, Cao H, Sun L, Dong S, Bian Y, Han J, Zhang L, Ren S, $\mathrm{Hu}$ Y, Liu C, et al: Antitumor activities of kushen: Literature review. Evid Based Complement Alternat Med 2012: 373219, 2012.

17. Yamazaki M: The pharmacological studies on matrine and oxymatrine. Yakugaku Zasshi 120: 1025-1033, 2000 (In Japanese).

18. Jiang G, Liu X, Wang M, Chen H, Chen Z and Qiu T: Oxymatrine ameliorates renal ischemia-reperfusion injury from oxidative stress through Nrf2/HO-1 pathway. Acta Cir Bras 30: 422-429, 2015.

19. Liu L, Lu W, Ma Z and Li Z: Oxymatrine attenuates bleomycininduced pulmonary fibrosis in mice via the inhibition of inducible nitric oxide synthase expression and the TGF- $\beta /$ Smad signaling pathway. Int J Mol Med 29: 815-822, 2012.

20. Zhang TZ, Fu Q, Chen T and Ma SP: Anti-asthmatic effects of oxymatrine in a mouse model of allergic asthma through regulating CD40 signaling. Chin J Nat Med 13: 368-374, 2015.

21. Zhao P, Zhou R, Li HN, Yao WX, Qiao HQ, Wang SJ, Niu Y, Sun T, Li YX and Yu JQ: Oxymatrine attenuated hypoxic-ischemic brain damage in neonatal rats via improving antioxidant enzyme activities and inhibiting cell death. Neurochem Int 89: 17-27, 2015.
22. Liu Y, Bi T, Dai W, Wang G, Qian L, Gao Q and Shen G: Effects of oxymatrine on the proliferation and apoptosis of human hepatoma carcinoma cells. Technol Cancer Res Treat: May 24, 2015 (Epub ahead of print)

23. Cohen SM: Cell proliferation and carcinogenesis. Drug Metab Rev 30: 339-357, 1998.

24. Suda T: Physiological and pathological roles of apoptosis. Nihon Rinsho 63 (Suppl 4): 395-400, 2005.

25. Zörnig M, Hueber A, Baum W and Evan G: Apoptosis regulators and their role in tumorigenesis. Biochim Biophys Acta 1551: F1-F37, 2001.

26. Cheng YM, Chou CY, Hsu YC, Chen MJ and Wing LY: The role of human papillomavirus type $16 \mathrm{E} 6 / \mathrm{E} 7$ oncoproteins in cervical epithelial-mesenchymal transition and carcinogenesis. Oncol Lett 3: 667-671, 2012.

27. Hamada K, Shirakawa T, Gotoh A, Roth JA and Follen M: Adenovirus-mediated transfer of human papillomavirus $16 \mathrm{E} 6 /$ E7 antisense RNA and induction of apoptosis in cervical cancer. Gynecol Oncol 103: 820-830, 2006.

28. Zhou J, Peng C, Li B, Wang F, Zhou C, Hong D, Ye F, Cheng X, Lü W and Xie X: Transcriptional gene silencing of HPV16 E6/ E7 induces growth inhibition via apoptosis in vitro and in vivo. Gynecol Oncol 124: 296-302, 2012.

29. Wu XN and Wang GJ: Experimental studies of oxymatrine and its mechanisms of action in hepatitis B and C viral infections. Chin J Dig Dis 5: 12-16, 2004.

30. Yao $\mathrm{N}$ and Wang $\mathrm{X}$ : In vitro immunomodulatory activity of oxymatrine on Toll-like receptor 9 signal pathway in chronic hepatitis B. Am J Chin Med 42: 1399-1410, 2014.

31. Lin M, Yang LY, Li WY, Peng YP and Zheng JK: Inhibition of the replication of hepatitis B virus in vitro by oxymatrine. J Int Med Res 37: 1411-1419, 2009. 